\title{
Techniques for the suppression of sidelobes in a non-contiguous orthogonal frequency division multiplexing framework
}

\author{
Atif Elahi $^{{ }^{*}}$, ljaz Mansoor Qureshi ${ }^{2,3}$, Fawad Zaman $^{4}$, Fahad Munir $^{1}$ and Adnan Umar ${ }^{1}$
}

*Correspondence:
atif.phdee40@iiu.edu.pk
${ }^{1}$ Department of Electronic
Engineering, Faculty
of Engineering
and Technology, International
Islamic University,
Islamabad 44000, Pakistan
Full list of author information
is available at the end of the
article

${ }^{*}$ Correspondence: atif.phdee40@iiu.edu.pk Engineering, Faculty of Engineering and Technology, International Islamic University, Full list of author information article

\begin{abstract}
In orthogonal frequency division multiplexing (OFDM)-based cognitive radio (CR) systems, high out-of-band radiation (OOBR) results in severe interference to the neighboring users, including licensed users (LUs) and cognitive radio users (CRUs). To tackle that issue, a framework of non-contiguous orthogonal frequency division multiplexing (NCOFDM) is presented in this paper that has the ability of describing any OOBR reduction techniques, regardless of whether a single or multiple techniques are applied. Based on this framework, we proposed four new different schemes that can be viewed as two-level suppression schemes. In the first level, the OOBR is reduced by techniques represented by precoding matrices, while in the second level further reduction of OOBR is done by using generalized sidelobe canceller (GSC). Numerical results show that the proposed schemes can suppress the OOBR significantly in terms of power spectral density (PSD), thus allowing the successful coexistence of LUs, as well as CRUs in a spectrum-sharing environment.
\end{abstract}

Keywords: Cognitive radio, Generalized sidelobe canceller, Non-contiguous orthogonal frequency division multiplexing, Out-of-band radiation, Power spectral density

\section{Background}

Orthogonal frequency division multiplexing (OFDM) is considered as the best candidate for cognitive radio systems (CRS) due to its capability of dividing the existing wideband channel into several narrow band orthogonal channels/subcarriers and to transmit those subcarriers in parallel. Some features of OFDM include spectral efficiency, multipath delay spread, robustness to channel fading, etc. On the other hand, due to the large sidelobes of the OFDM subcarriers, CR based on OFDM experiences high out-ofband radiation (OOBR) (Goldsmith 2005; Proakis 2008) that may result in considerable interference with the adjacent bands used by either LUs or CRUs.

To overcome this issue, a number of methods have been proposed recently that can be divided into two categories. Methods belonging to the first category are called the time domain methods, including filtering (Noguet et al. 2011), windowing (El-Saadany et al. 2009; Sahin and Arslan 2011), etc., where the OOBR reduction process is carried out after the inverse fast Fourier transformation (IFFT). Methods belonging to the second

(c) 2016 The Author(s). This article is distributed under the terms of the Creative Commons Attribution 4.0 International License (http://creativecommons.org/licenses/by/4.0/), which permits unrestricted use, distribution, and reproduction in any medium, provided you give appropriate credit to the original author(s) and the source, provide a link to the Creative Commons license, and indicate if changes were made. 
category are called the frequency domain methods, including subcarrier weightings (SW) (Cosovic et al. 2006), advanced subcarrier weightings (ASW) (Selim and Doyle 2013), cancellation carriers (CC) (Brandes et al. 2005; Pagadarai et al. 2008b), advanced cancellation carriers (ACC) (Selim et al. 2013; Rady et al. 2015), CC using (genetic algorithm) GA and (differential evolution) DE (Elahi et al. 2016), active and null cancellation carriers (ANCC) (Lopes and Panaro 2013), constellation expansion (CE) (Pagadarai et al. 2008a; Li et al. 2009; Selim et al. 2012), CE using GA (Khan and Yoo 2015) and generalized sidelobe canceller (GSC) (Elahi et al. 2015), etc. where the OOBR elimination process is carried out before the IFFT. Since the available methods suffer from several drawbacks, a suitable combination of two or more than two methods might improve the overall OOBR reduction and alleviate their drawbacks.

In this paper, a non-contiguous orthogonal frequency division multiplexing (NCOFDM) framework is presented that has the ability of representing any OOBR reduction scheme, regardless of whether individual or multiple schemes are applied. Then, according to the place where different schemes are applied, they are categorized into three groups, namely, symbol mapping, precoding and times domain techniques. Based on that framework, four new different schemes are introduced that can be viewed as two-level suppression schemes. In the first level, the OOBR is reduced using CC (Brandes et al. 2005), CC (Pagadarai et al. 2008b), ACC (Selim et al. 2013) and ANCC (Lopes and Panaro 2013), while in the second level, further reduction of OOBR is achieved using GSC. The performance of the proposed schemes is shown with the help of simulations, which show a better reduction of OOBR as compared to the results of the existing methods.

The rest of the paper is organized as follows: the first section describes the "System model", followed by a description of the "Proposed techniques". "Simulations results" provides the simulation results of the proposed schemes, while the paper ends with the "Conclusion".

Notation: $(.)^{H},(.)^{T},()^{*}$ and $(.)^{-1}$ represent the Hermitian, Transpose, conjugate and inverse, while $\mathrm{E}[$.$] represents the expectation.$

The lowercase bold letter represents a vector, the uppercase bold letter the matrix, I the identity matrix and $\mathbf{O}$ the null matrix.

\section{System model}

The block diagram of a non-contiguous orthogonal frequency division multiplexing (NCOFDM) framework is given in Fig. 1.

Consider " $M$ " cognitive radio users (CRUs) also called as unlicensed users that employ the available spectrum opportunistically with the help of spectrum sensing. The employed spectrum may be interrupted or uninterrupted, divided into a group of subcarriers $\beta=\left[n_{0}, n_{1}, \ldots, n_{N-1}\right]$. Here the $k t h$ CRU, $0 \leq k \leq K-1$, employs the subgroup of subcarriers $\beta_{k} \in \beta$, where $\beta_{k}=\left[n_{0}^{k}, n_{1}^{k}, \ldots, n_{N_{k}-1}^{k}\right]$ consists of $N_{k}$ subcarriers in total. Suppose $g_{n}$ represents the transmitted symbol over the $n$th subcarrier. The baseband signal over the $n$th subcarrier with one symbol duration is given as:

$$
x(t)=\sum_{n=0}^{N-1} g_{n} e^{j 2 \pi \frac{n}{T_{s}} t} w(t),
$$




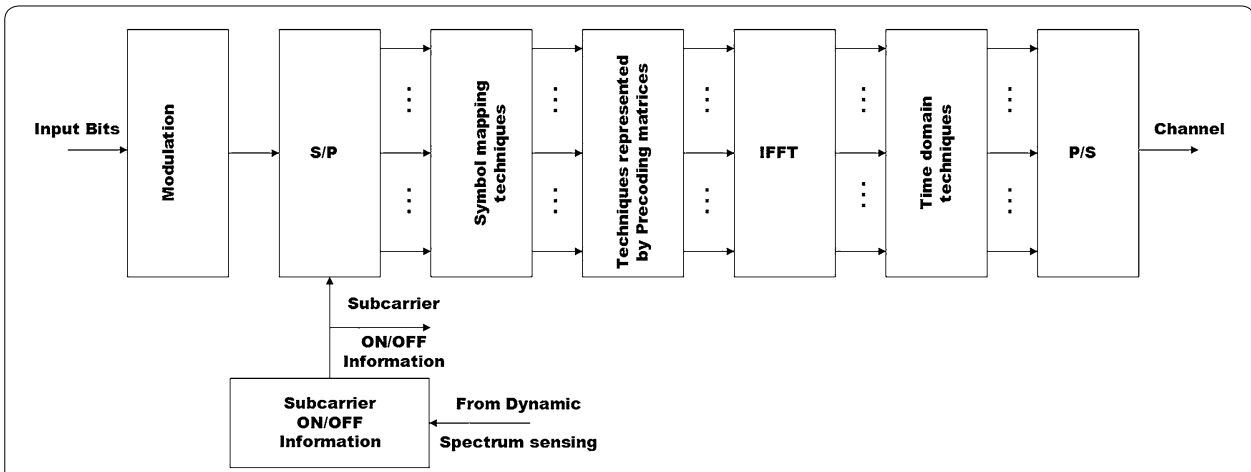

Fig. 1 Framework of non-contiguous OFDM transmitter

where $T_{s}$ is the duration of a symbol, while $w(t)$ is the windowing function that can be written as:

$$
w(t)=\left\{\begin{array}{l}
1-T_{g} \leq t \leq T_{s} \\
0 \quad \text { otherwise }
\end{array}\right.
$$

where $T_{g}$ the length of the cyclic prefix is inserted to eliminate the influence of intersymbol interference (ISI).

After taking the Fourier transformation of Eq. (1), the NCOFDM signal in the frequency domain is given as:

$$
X(f)=\sum_{n=0}^{N-1} g_{n} s_{n}(f),
$$

where

$$
s_{n}(f)=\mathrm{Te}^{-j \pi\left(T_{s}-T_{g}\right)\left(f-\frac{n}{T_{s}}\right)} \sin c\left(T\left(f-\frac{n}{T_{s}}\right)\right) .
$$

Here, $T$ is defined as the symbol duration; with the help of Eqs. (3) and (4) we can get the power spectral density (PSD) of the NCOFDM signal as:

$$
\begin{aligned}
& P(f)=\frac{1}{T} E\left\{|X(f)|^{2}\right\}, \\
& P(f)=\frac{1}{T} \mathbf{s}^{T}(f) E\left(\mathbf{g g}^{H}\right) \mathbf{s}^{*}(f) .
\end{aligned}
$$

Here, $\mathbf{s}(f)=\left[s_{n_{0}}(f), s_{n_{1}}(f), \ldots, s_{n_{N-1}}(f)\right]^{T}$ and $\mathbf{g}=\left[g_{n_{0}}, g_{n_{1}}, \ldots, g_{n_{N-1}}\right]^{T}$.

Now to protect the LUs which are present in the neighboring frequency bands of the CRUs, the PSD of the NCOFDM signal should be minimized where the LUs are present.

\section{Proposed techniques}

In this section, we present four schemes, each having two levels to minimize the OOBR to protect the LUs from the interference of the CRUs. In the first level, the OOBR is minimized using methods which are represented by precoding matrices; these include 
CC (Brandes method) (Brandes et al. 2005), CC (Pagadarai method) (Pagadarai et al. 2008b), ACC (Selim et al. 2013) and ANCC (Lopes and Panaro 2013), while in the second level further minimization of OOBR is achieved using GSC (Elahi et al. 2015).

\section{First-level minimization}

As shown in Fig. 1, the input bit stream is first modulated with phase shift keying (PSK) or quadrature amplitude modulation (QAM) and then passed through the serial to parallel (S/P) block. Consider $\mathbf{b}_{k}=\left[b_{k}(0), b_{k}(1), \ldots, b_{k}\left(N_{k}-1\right)\right]^{T}$ that represents the data symbols for the $k$ th CRU, where $b_{k}(j)$ represents the $j$ th symbol of the $k$ th CRU. Assuming that the information symbols are independently and identically distributed (iid):

$$
E\left[\mathbf{b}_{k} \mathbf{b}_{k}^{H}\right]=\mathbf{I}_{N_{k} \times N_{k}},
$$

where I represents the identity matrix with dimension $N_{k} \times N_{k}$. After S/P, the data symbol vector is then taken to the frequency domain approach block, the output of which is given by:

$$
\mathbf{d}_{k}=\mathbf{Q}_{k} \mathbf{b}_{k},
$$

where $\mathbf{d}_{k}=\left[d_{k}(0), d_{k}(1), \ldots, d_{k}\left(N_{d}-1\right)\right]^{T}$ represents the precoded symbol vector for the $k$ th CRU (note that the dimension of $\mathbf{d}_{k}$ is greater than $\left.\mathbf{b}_{k}\right), M_{k}=\left(N_{d}-N_{k}\right)$ is the total number of CCs and

$$
\mathbf{Q}_{k}=\left[\begin{array}{c}
\mathbf{A}_{\frac{M_{k}}{2} \times N_{k}} \\
\mathbf{I}_{N_{k} \times N_{k}} \\
\mathbf{B}_{\frac{M_{k}}{2} \times N_{k}}
\end{array}\right]
$$

can be viewed as a precoding matrix with dimension $N_{d} \times N_{k}$ representing the CC (Brandes method), CC (Pagadarai method), ANCC and ACC which deactivate the subcarriers on the edges and calculate the amplitudes of these subcarriers within defined threshold power that reduces the OOBR in the specific frequency range where LUs are operating. The identity matrix I in Eq. (9) represents the weighting coefficients of the data subcarriers, while the two matrices $\mathbf{A}$ and $\mathbf{B}$ in Eq. (9) represent the optimized values of the left- and right-sided CCs. The resulting signal for the $k$ th CRU after first-level minimization in the frequency domain will be then given by:

$$
T_{k}(f)=\sum_{j=1}^{\frac{M_{k}}{2}} d_{k, j} s_{k, j}(f)+\sum_{n=0}^{N_{k}-1} d_{k, n} s_{k, n}(f)+\sum_{j=\frac{M_{k}}{2}+1}^{M_{k}} d_{k, j} s_{k, j}(f) .
$$

The PSD of the signal of the $k$ th CRU after first-level minimization is given by:

$$
P_{k}(f)=\frac{1}{T} E\left\{\left|T_{k}(f)\right|^{2}\right\}=\frac{1}{T} \mathbf{s}_{k}^{T}(f) E\left\{\mathbf{d}_{k} \mathbf{d}_{k}^{H}\right\} \mathbf{s}_{k}^{*}(f) .
$$

\section{Second-level minimization}

In the second-level minimization, further reduction of OOBR is done by first taking the $W$ samples of the signal given in Eq. (10) and are collected in vector $\mathbf{z}_{k}$ having dimension 
$W \times 1$ and passed through the GSC (Elahi et al. 2015). A frequency domain approach is shown in Fig. 2.

GSC is the simplest version of linearly constraint minimum variance (LCMV), where the constraint minimization problem is converted into an unconstraint minimization problem and has two branches. The vector $\mathbf{u}_{q}$ in the upper branch, also called the quiescent weight vector $\mathbf{u}_{a}$, is not adaptive but preserves the coming signal, whereas the lower branch has a blocking matrix $\mathbf{B}$ followed by an adaptive weight vector. The purpose of blocking matrix $\mathbf{B}$ is to block the chosen part of the signal and maintain the rest of the part. The output of the GSC is given by:

$$
\begin{aligned}
& Y_{k}(f)=\mathbf{u}_{q}^{H} \mathbf{z}_{k}-\mathbf{u}_{a}^{H} \mathbf{B} \mathbf{z}_{k}, \\
& Y_{k}(f)=\left(\mathbf{u}_{q}^{H}-\mathbf{u}_{a}^{H} \mathbf{B}\right) \mathbf{z}_{k} .
\end{aligned}
$$

The adaptive weight vector $\mathbf{u}_{a}$ is used to minimize the sidelobes, which result in OOBR. It adjusts the amplitudes of sidelobes, such that when the signals from the upper and lower branch add with each other, a signal with minimum OOBR results. The expressions for $\mathbf{u}_{q}, \mathbf{u}_{a}$ and $\mathbf{B}$ are available in Elahi et al. (2015) and are given by:

$$
\begin{aligned}
& \mathbf{u}_{q}=\mathbf{C}\left(\mathbf{C}^{H} \mathbf{C}\right)^{-1} \mathbf{g}, \\
& \mathbf{B}=\operatorname{null}\left(\mathbf{C}^{H}\right) .
\end{aligned}
$$

The optimized adaptive weight vector $\mathbf{u}_{a(o p t)}$ is the one that minimizes the cost function

$$
J\left(\mathbf{u}_{a}\right)=\left(\mathbf{u}_{q}-\mathbf{B} \mathbf{u}_{a}\right)^{H} \mathbf{R}_{\mathbf{z}}\left(\mathbf{u}_{q}-\mathbf{B} \mathbf{u}_{a}\right)
$$

i.e., $\min _{\mathbf{u}_{a}}\left(\mathbf{u}_{q}-\mathbf{B} \mathbf{u}_{a}\right)^{H} \mathbf{R}_{\mathbf{z}}\left(\mathbf{u}_{q}-\mathbf{B} \mathbf{u}_{a}\right)$ and on solving gives:

$$
\mathbf{u}_{a(o p t)}=\left(\mathbf{B}^{H} \mathbf{R}_{\mathbf{z}} \mathbf{B}\right)^{-1} \mathbf{B}^{H} \mathbf{R}_{\mathbf{z}} \mathbf{u}_{q}
$$

Finally,

$$
\mathbf{u}_{G S C}=\mathbf{u}_{q}-\mathbf{B} \mathbf{u}_{a(o p t)} .
$$

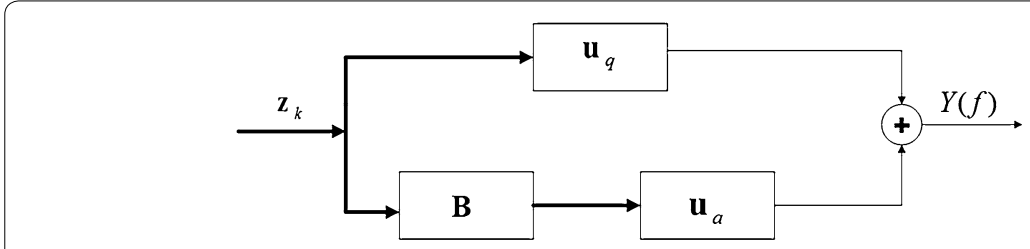

Fig. 2 Block diagram of GSC 


\section{Simulation results}

This section is divided into two portions. In the first portion, the simulation results demonstrated the performance of the proposed schemes for single CRU operating in the vacant space. The number of subcarriers utilized by a single CRU is 16, in which, e.g., a 64-point FFT is applied for OFDM modulation. Every subcarrier is modulated with a BPSK symbol whose power is normalized to 1 . For the first-level suppression in all four proposed schemes, two CCs are reserved on both sides of the data subcarriers. Ten sidelobes are taken on both sides of the spectrum with one frequency sample taken in the middle of every sidelobe in the optimization range, resulting in $K_{1}=K_{\mathrm{r}}=10$. For the second-level suppression, 301 samples are taken. In Figs. 3, 4, 5 and 6, the PSD curves representing the NCOFDM signals of single CRU utilizing the subband are shown.

In the second portion, we present the simulation results to represent different CRUs operating in a non-contiguous band. In Figs. 7, 8, 9 and 10, the PSD curves of four CRUs signal in a non-contiguous band have subcarriers $\{88, \ldots, 120,200, \ldots, 232,320, \ldots, 352$, $437, \ldots, 469\}$, and 128-point FFT can be applied for NCOFDM modulation. Each subcarriers is modulated with BPSK symbol whose power is normalized to 1 . The spacing between each CRU is considered as equal. In the first-level suppression, two CCs are

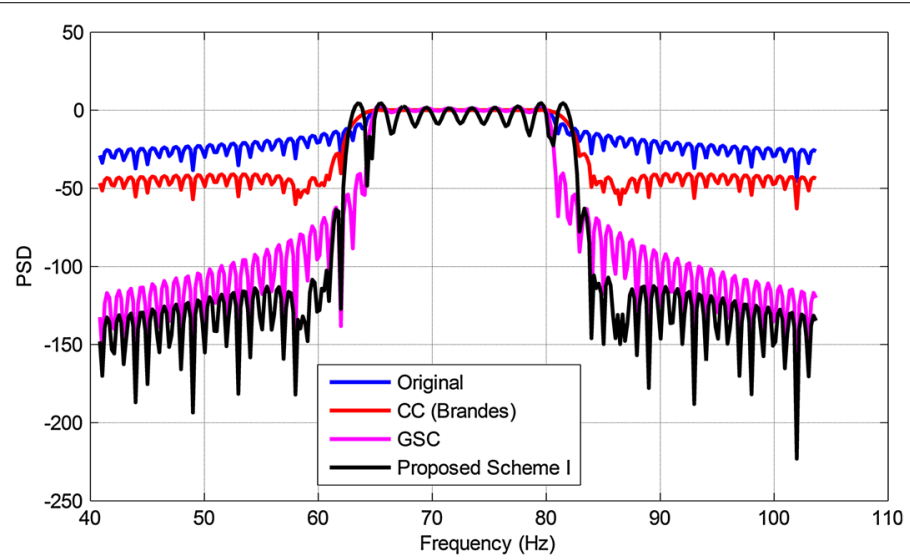

Fig. 3 PSD curve of single CRU with the proposed scheme I

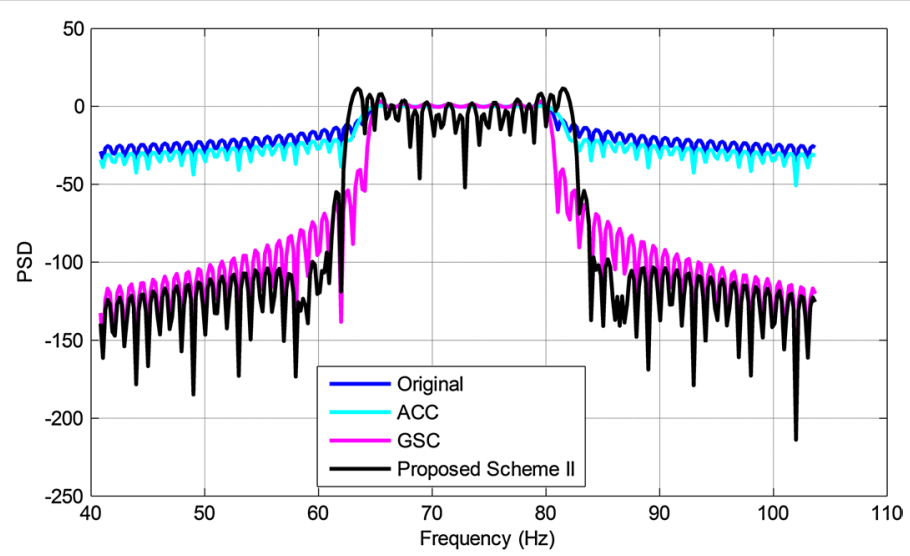

Fig. 4 PSD curve of single CRU with the proposed scheme II 


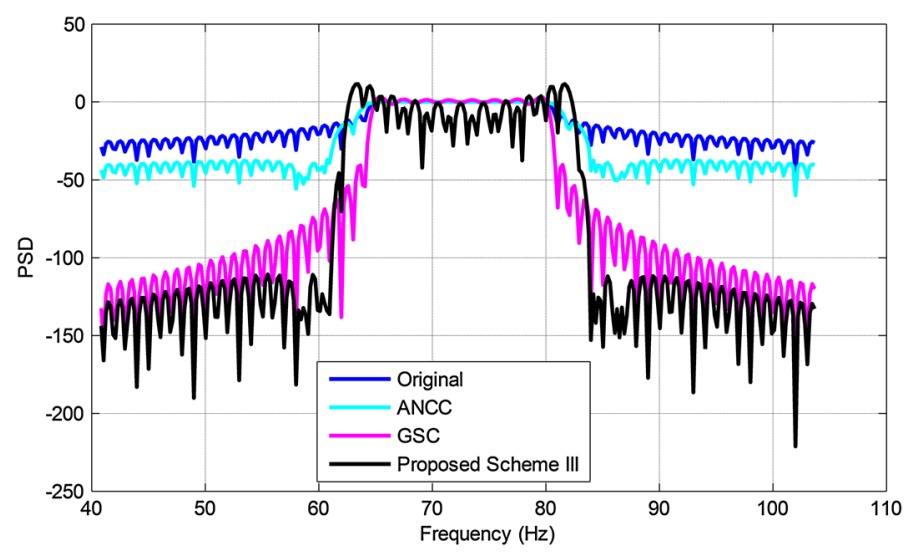

Fig. 5 PSD curve of single CRU with the proposed scheme III

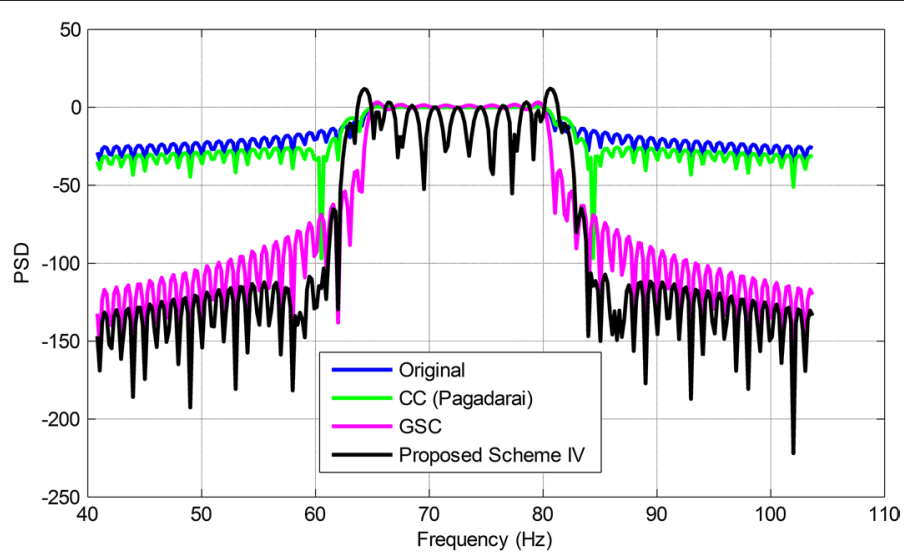

Fig. 6 PSD curve of single CRU with the proposed scheme IV

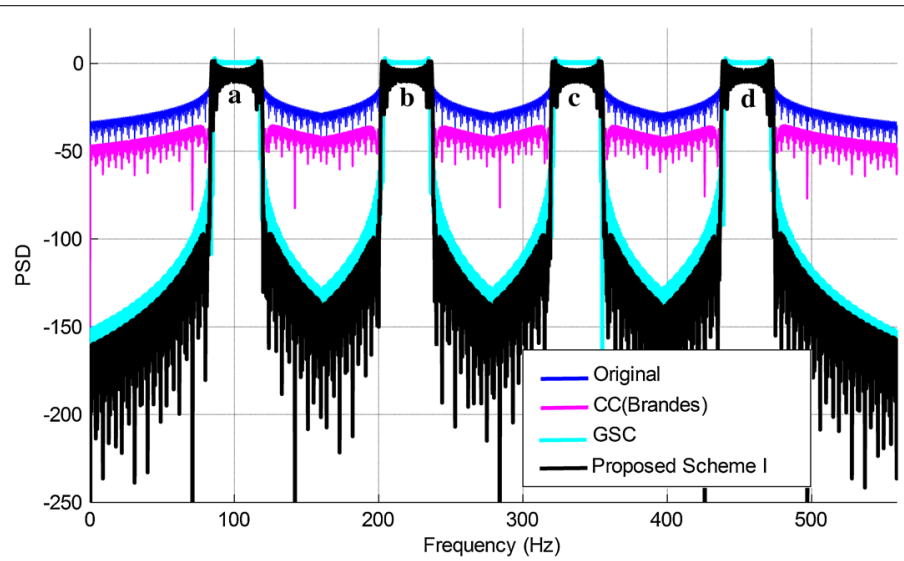

Fig. 7 PSD curve of different CRUs having equal spacing with the proposed scheme I

considered on either side of the subcarriers used by the CRUs, and ten sidelobes on either side of the used spectrum in the optimization range is considered in all four proposed schemes. In the second-level suppression, 766 samples are considered. 


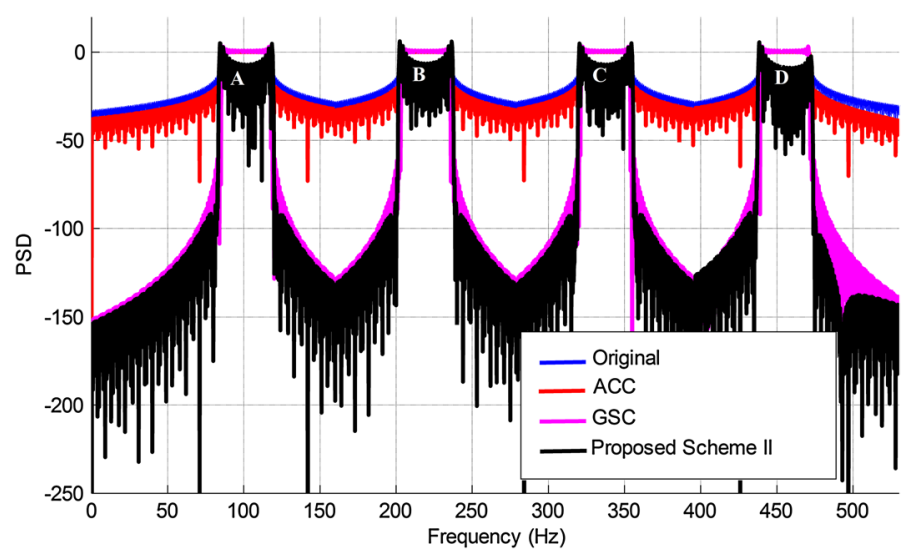

Fig. 8 PSD curve of different CRUs having equal spacing with the proposed scheme II

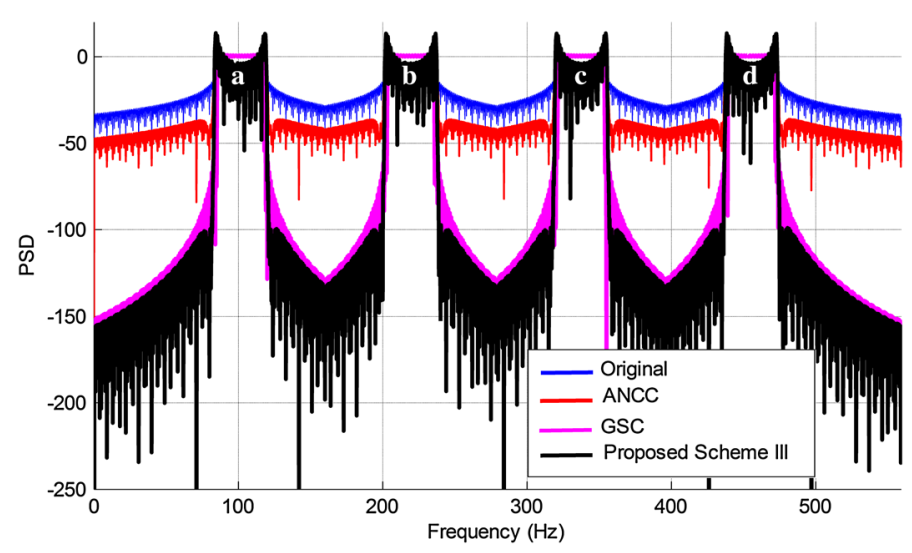

Fig. 9 PSD curve of different CRUs having equal spacing with the proposed scheme III

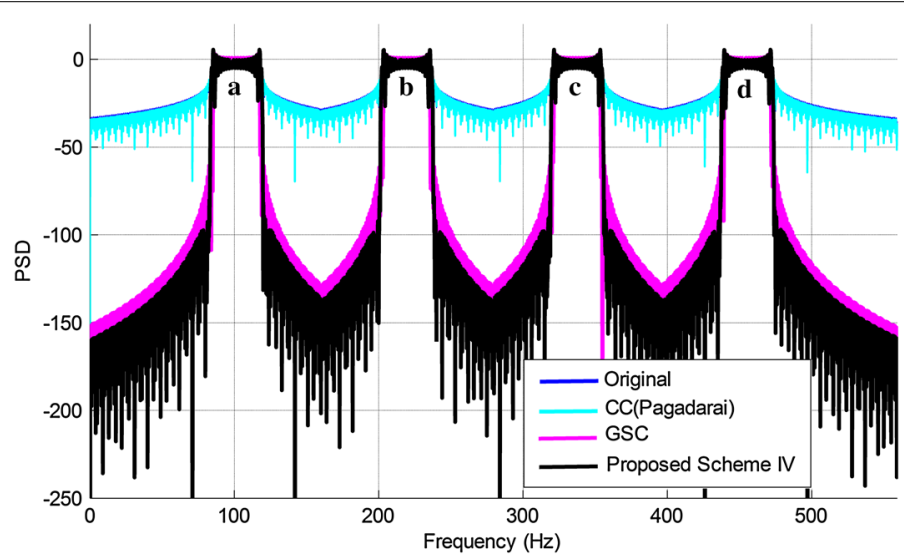

Fig. 10 PSD curve of different CRUs having equal spacing with the proposed scheme IV

In Figs. 11, 12, 13 and 14, the PSD curves of four portions of CRUs signal in a noncontiguous band consisting of subcarriers $\{61, \ldots, 93,197, \ldots, 229,320, \ldots, 352,470, \ldots, 502\}$ are shown. The spacing between them is considered as unequal, and 128-point FFT is 


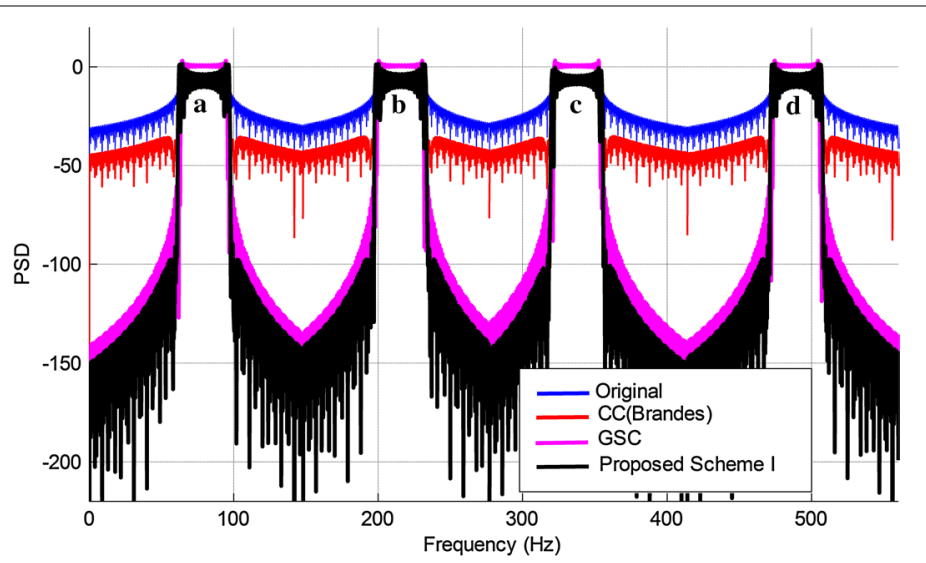

Fig. 11 PSD curves of four CRUs signals having unequal spacing with the proposed scheme I

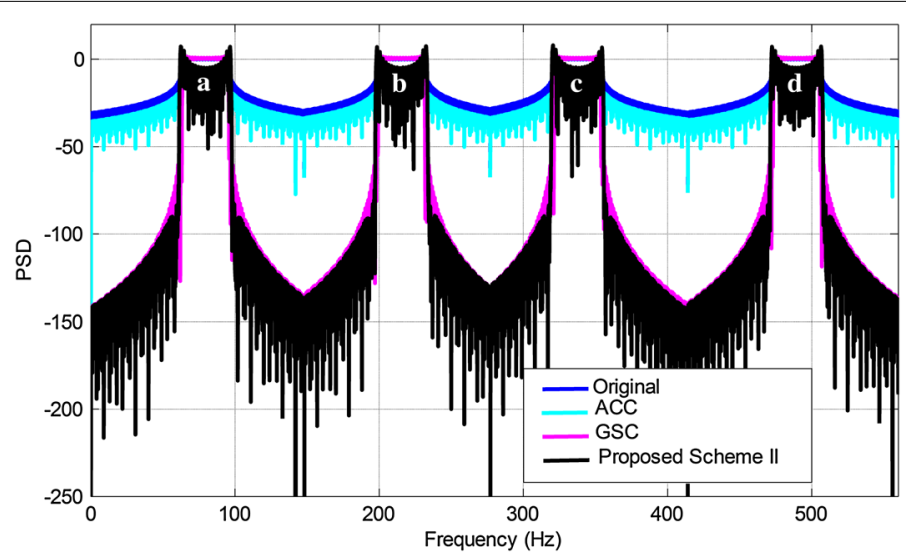

Fig. 12 PSD curves of four CRUs signals having unequal spacing with the proposed scheme II

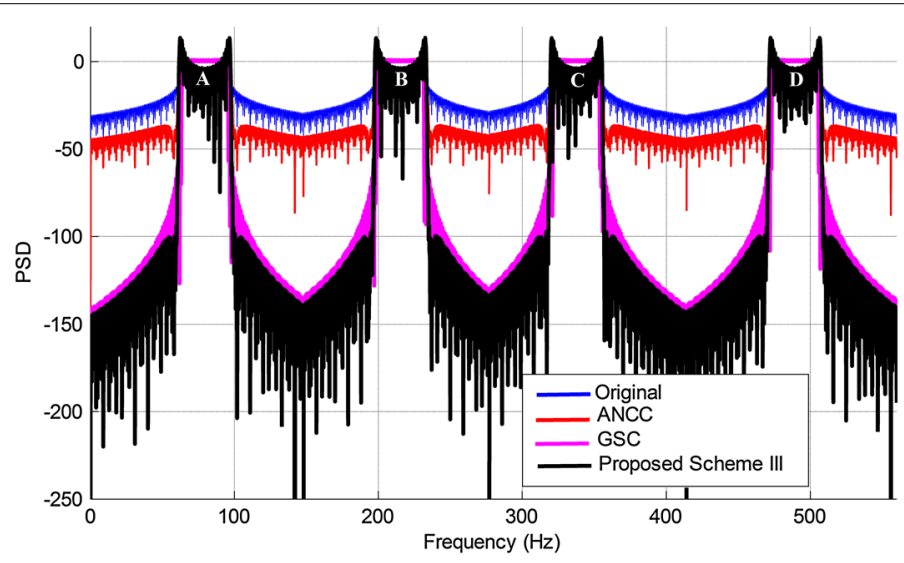

Fig. 13 PSD curves of four CRUs signals having unequal spacing with the proposed scheme III

applied for NCOFDM modulation and each subcarrier is modulated with BPSK with normalized power equal to 1 . In all the proposed schemes in first-level suppression, two CCs are taken on either side of the used subcarriers and ten sidelobes are taken 


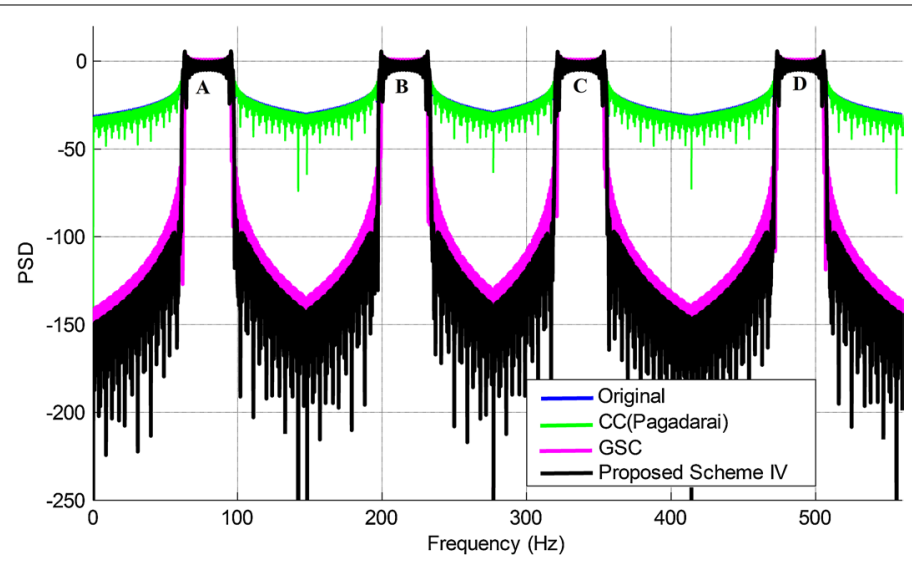

Fig. 14 PSD curves of four CRUs signals having unequal spacing with the proposed scheme IV

as samples in the optimization range. In the second-level suppression, 707 samples are taken.

It can be shown from Figs. 3, 4, 5, 6, 7, 8, 9, 10, 11, 12, 13 and 14 that the resulting PSD curves of the proposed schemes are well below the PSD curves of the existing methods, and sufficient OOBR reduction is achieved in a single user as well as in a multi-CRU environment.

\section{Conclusion}

In this paper, we have proposed four different two-level suppression schemes for the reduction of OOBR in NCOFDM-based single as well as multi-CRU environments. For this purpose, we present the NCOFDM framework that has the capability of describing any OOBR suppression schemes, no matter whether we are applying a single or multiple schemes. In this framework, according to the location where the OOBR suppression schemes are employed, these are divided into three groups, which are symbol mapping, precoding and time domain methods. Based on this NCOFDM framework, four, twolevel OOBR suppression schemes are proposed, which are the combinations of GSC with the other four methods represented by precoding matrices, namely CC (Brandes et al. 2005), CC (Pagadarai et al. 2008b), ACC (Selim et al. 2013) and ANCC (Lopes and Panaro 2013). Numerical results show that all the proposed schemes get significant reduction of OOBR in a spectrum-sharing environment.

\section{Authors' contributions}

AE proposed and implemented the idea. IMQ and FZ supervised. AU and FM helped in drafting and writing of this manuscript. All authors read and approved the final manuscript.

\section{Author details}

'Department of Electronic Engineering, Faculty of Engineering and Technology, International Islamic University, Islamabad 44000, Pakistan. ${ }^{2}$ Department of Electrical Engineering, Air University, Islamabad 44000, Pakistan. ${ }^{3}$ Institute of Signal Systems and Soft Computing (ISSS), Islamabad 44000, Pakistan. ${ }^{4}$ Department of Electrical Engineering, COMSATS Institute of Information Technology, Attock Campus, Attock 43600, Pakistan.

\section{Acknowledgements}

The authors thank Miss. Mehreen for her assistance and support of this research.

\section{Competing interests}

The authors declare that they have no competing interests. 
Received: 4 April 2016 Accepted: 17 August 2016

Published online: 26 August 2016

\section{References}

Brandes S, Cosovic I, Schnell M (2005) Sidelobe suppression in OFDM systems by insertion of cancellation carriers. In: IEEE 62nd vehicular technology conference, VTC-2005-Fall. IEEE. pp 152-156

Cosovic I, Brandes S, Schnell M (2006) Subcarrier weighting: a method for sidelobe suppression in OFDM systems. Commun Lett IEEE 10:444-446

Elahi A, Qureshi IM, Khan ZU, Zaman F (2015) Sidelobe reduction in non-contiguous OFDM-based cognitive radio systems using a generalized sidelobe canceller. Appl Sci 5:894-909

Elahi A, Qureshi IM, Zaman F, Munir F (2016) Reduction of out of band radiation in non-contiguous OFDM based cognitive radio system using heuristic techniques. J Inf Sci Eng 32:349-364

El-Saadany MS, Shalash AF, Abdallah M (2009) Revisiting active cancellation carriers for shaping the spectrum of OFDMbased cognitive radios. In: IEEE sarnoff symposium, SARNOFF'09. IEEE, pp 1-5

Goldsmith A (2005) Wireless communications. Cambridge University Press, Cambridge

Khan H, Yoo S-J (2015) Dynamic interference control in OFDM-based cognitive radio network using genetic algorithm

Li D, Dai X, Zhang H (2009) Sidelobe suppression in NC-OFDM systems using constellation adjustment. Commun Lett IEEE 13:327-329

Lopes FRB, Panaro JSG (2013) OFDM sidelobe suppression combining active and null cancellation carriers in the guard bands. In: Microwave and optoelectronics conference (IMOC), SBMO/IEEE MTT-S International. IEEE, pp 1-5

Noguet D, Gautier M, Berg V (2011) Advances in opportunistic radio technologies for TVWS. EURASIP J Wirel Commun Netw 2011:1-12

Pagadarai S, Rajbanshi R, Wyglinski AM, Minden GJ (2008a) Sidelobe suppression for OFDM-based cognitive radios using constellation expansion. In: IEEE wireless communications and networking conference, WCNC 2008. IEEE, pp 888-893

Pagadarai S, Wyglinski AM, Rajbanshi R (2008b) A sub-optimal sidelobe suppression technique for OFDM-based cognitive radios. In: IEEE military communications conference, MILCOM 2008. IEEE, pp 1-6

Proakis JG, Salehi M (2008) Digital Communications. McGraw-Hill, New York, NY

Rady A, Shokair M, El-Rabaie E-SM, ElKorany AS (2015) Efficient technique for sidelobe suppression and PAPR reduction in OFDM-based cognitive radios. Wirel Pers Commun 83:215-230

Sahin A, Arslan H (2011) Edge windowing for OFDM based systems. Commun Lett IEEE 15:1208-1211

Selim A, Doyle L (2013) Real-time sidelobe suppression for OFDM systems using advanced subcarrier weighting. In: IEEE wireless communications and networking conference (WCNC). IEEE, pp 4043-4047

Selim A, Ozgul B, Doyle L (2012) Efficient sidelobe suppression for OFDM systems with peak-to-average power ratio reduction. In: IEEE international symposium on dynamic spectrum access networks (DYSPAN) 2012. IEEE, pp 510-516

Selim A, Macaluso I, Doyle L (2013) Efficient sidelobe suppression for OFDM systems using advanced cancellation carriers. In: IEEE international conference on communications (ICC). IEEE, pp 4687-4692

\section{Submit your manuscript to a SpringerOpen ${ }^{\circ}$ journal and benefit from:}

Convenient online submission

- Rigorous peer review

- Immediate publication on acceptance

- Open access: articles freely available online

- High visibility within the field

- Retaining the copyright to your article

Submit your next manuscript at $>$ springeropen.com 